\title{
Deinococcus Radiodurans: The World's Toughest Bacterium. A Review
}

By Dr. Sujan Narayan Agrawal, Satyaram Satpathy \& Debashish Samal Abstract- Deinococcus Radiodurans is the "world's toughest bacterium" as per the Guinness book of world record. It is the most radiation-resistant bacterium ever known. It can withstand severe dehydration, cold, vacuum, acid, lack of nutrition, and survive to the radiation dose, a fraction of which is sufficient to kill the human being. The meaning of its name is 'strange berry that withstands radiation.' This remarkable talent is extensively studied by the biologist and scientist to find out how it survives the extreme life-threatening conditions. This knowledge is being used to find out the means to survive in radiation exposure and also to handle toxic waste.

Keywords: deinococcus radiodurans, extremophilic bacterium.

GJMR-C Classification: NLMC Code: QW 25.5.B2

Strictly as per the compliance and regulations of:

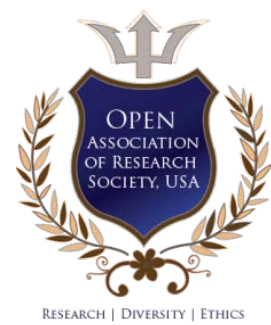

(c) 2020. Dr. Sujan Narayan Agrawal, Satyaram Satpathy \& Debashish Samal. This is a research/review paper, distributed under the terms of the Creative Commons Attribution-Noncommercial 3.0 Unported License http://creativecommons.org/licenses/by$\mathrm{nc} / 3.0 /$ ), permitting all non-commercial use, distribution, and reproduction in any medium, provided the original work is properly cited. 


\title{
Deinococcus Radiodurans: The World's Toughest Bacterium. A Review
}

\author{
Dr. Sujan Narayan Agrawal ${ }^{\alpha}$, Satyaram Satpathy ${ }^{\sigma} \&$ Debashish Samal ${ }^{\rho}$
}

\begin{abstract}
Deinococcus Radiodurans is the "world's toughest bacterium" as per the Guinness book of world record. It is the most radiation-resistant bacterium ever known. It can withstand severe dehydration, cold, vacuum, acid, lack of nutrition, and survive to the radiation dose, a fraction of which is sufficient to kill the human being. The meaning of its name is 'strange berry that withstands radiation.' This remarkable talent is extensively studied by the biologist and scientist to find out how it survives the extreme life-threatening conditions. This knowledge is being used to find out the means to survive in radiation exposure and also to handle toxic waste.
\end{abstract}

Keywords: deinococcus radiodurans, extremophilic bacterium.

\section{INTRODUCTION}

rastir he name Deinococcus is derived from Greek, the dinos, meaning strange or unusual, and coccus, meaning a "terrible grain/berry", and in the Latin radius and durare, meaning "radiation surviving"[1] It is a Gram-positive, red-pigmented, nonsporulating, non- pathogenic Bacteria occurring in diads and tetrads with an average cell diameter of 1 m (range, 0.5 to 3.5 m). [2] It is an Extremophilic bacterium. It is one of the most radiation-resistant organisms ever known to humanity. It can survive extreme cold, dehydration, vacuum and, acid. It is therefore known as a polyextremophilic bacterium. [3] In the Gunnies Book of World records it is listed as the toughest bacterium. It belongs to Genus Deinococcus, the type species is radiodurans; The other known members of this genus are $\mathrm{D}$. proteolyticus, D. radiopugnans, D. radiophilus, D. grandis, D. indicus, D. frigens, D. saxicola, D. marmoris, D. deserti, D. Geothermalis, and D. Murrayi. [4] All Dienococcus species are distinguished by their extraordinary ability to tolerate the lethal effects of DNA-damaging agents, particularly those of ionizing radiation and UV radiation. $[5]$.

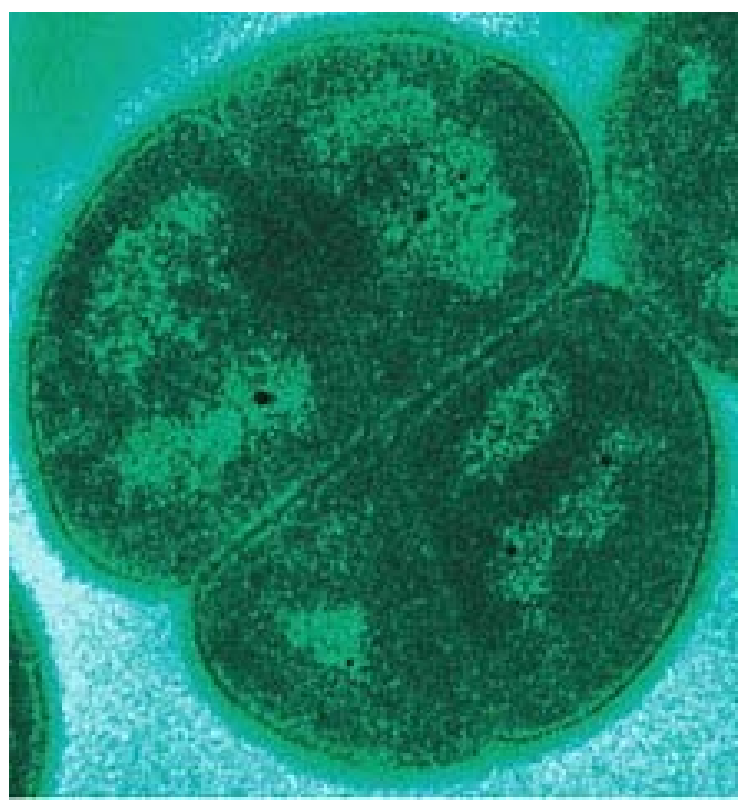

Figure 1: Deinococcus Radiodurans

How to cite this article:

Dr. Sujan Narayan Agrawal, Satyaram Satpathy \& Debashish Samal. Deinococcus Radiodurans: The World's Toughest Bacterium. A Review. GJMR(C) 2020; 20(2):pp 1-4.

DOI: https://doi.org/10.34257/GJMRCVOL20IS2PG1

Author a: Professor (Department of Surgery) SBRKM Government Medical College, Jagdalpur (Bastar) Chattisgarh, INDIA. 


\section{THE DiscOVERY}

D. radiodurans was discovered in 1956 by Arthur Anderson at the Oregon Agricultural Experiment Station in Corvallis Oregon. The discovery occurred during an experiment to find out, whether, canned food can be sterilized using gamma irradiation? In the experiment, a tin of meat was exposed to high doses of radiation sufficient to kill all the known life forms, but the meat was spoiled, and D. Radiodurans survived and isolated. [6]

The cell Stains gram-positive, although its cell envelop is unusual and is reminiscent of the cell walls of Gram-negative bacteria. It is due to its multilayered structure and lipid composition.D. Radiodurans is a spherical bacterium with a diameter of 1.5 to $3.5 \mu \mathrm{m}$. Four cells stick together, forming a tetrad. It is nonmotile and does not form endospores. It uses oxygen to derive energy from organic compounds in its environment. It is often found in habitats rich in organic materials, such as sewage, meat, feces, or soil. It is also isolated from the medical instruments, room dust, textile, and dried food. [5] It is extremely resistant to ionizing radiation, ultraviolet rays, desiccation, and electrophilic agents. [7]

\section{ili. The Genome}

The genome consists of two circular strips of chromosomes. It contains about 3195 genes. In the normal stationary phase, each bacterial cell has four copies of this genome. When they are rapidly multiplying, each bacterium may have 8-10 copies of the genome. It is capable of withstanding a dose of 5000 Grays (Gy) or 500,000 rad of ionizing radiation without any loss of viability. As compare to this, the human being can be killed by a radiation dose, as low as 5 Gy. [8-9]

It is a gram-positive nonsporulating bacterium that usually grows in a tetrad form. It is can survive the extreme radiation exposure due to its ability to repair the genome, without loss of genomic integrity or mutation. This remarkable feature is due to the presence of a robust DNA repair system that can accurately restore genomic integrity following the introduction of hundreds of double-stranded genomic breaks. (DSBs). [5] The Deinococcus radiodurans isolates the damaged segments in a controlled area and repairs it. These bacteria can also repair many small fragments from an entire chromosome. Its survival property from high ionizing radiation is due to the presence of multiple copies of its genome and its rapid DNA repair mechanism. The ionizing radiation brings several breaks in its genome. These breaks in chromosomes are repaired within 12-24 hours by a two-step process. It seems that several genome maintenance proteins work together to mediate the process of DNA repair. After the double-stranded breaks the genome reconstruction occurs in two phases:

1. In the first phase within one hour, a process called "extended synthesis-dependent single-stranded DNA annealing (ESDSA) resects DSB ends to produce $3^{\prime}$ single-stranded DNA (ssDNA) extensions. These ends are then paired with homologous duplex DNA to create templates for DNA synthesis by DNA polymerases.

2. In the second phase, which occurred after 1-2 hours of radiation damage RecA-mediated recombination, which requires removal of SSB and resolution of interlinked chromosomes, completes the repair process. [10]

It is interesting to note that this process does not introduce any more mutation than, a normal round of replication work. It is also capable of genetic transformation. It is a process in which DNA derived from one cell can be taken up by another cell and integrates into the recipient genome by homologous recombination. [11] "The organism can put its genome back together with absolute fidelity," says Claire $M$. Fraser, of The Institute for Genome Research (TIGR) in Rockville, Maryland. She was the leader of the TIGR team that sequenced D. radiodurans in 1999.

Michael Daly suggested that the bacterium uses Manganese complexes as antioxidants, thereby protecting itself from oxidative damages. [12]. Michael Daly of the Uniformed Services University of the Health Sciences in Bethesda, Maryland, and his team come up with a possible explanation. It is because this bacterium store a high level of manganese and relatively low levels of iron. It seen that bacteria which shrivel up with a dose of radiation have little manganese and more of Iron. Michael further suggests that the manganese helps to clean up the free radicals that are released by the bacterium during the metabolic process. This manganese store makes the bacterium healthie, $r$ and they are better equipped to mend the radiation damages. This theory is now tested by elevating the manganese levels of E.Coli bacteria. If the experiment and the said theory are proved then, it may help to prevent the radiation damage during chemotherapy in cancer patients. But it is too premature speculation since we still do not know that the survival of this bacterium from radiation damage is really due to high levels of manganese or otherwise. Michael has also suggested that "the protein, rather than the DNA is the principal target of the biological action of [lonizing radiation] in the bacteria. The extreme resistance in $\mathrm{Mn}$ accumulating bacteria is based on protein protection" [13]. M.Peana and C.Chasapis in 2018 proposed a model for Manganese interaction with the DR Proteome network involved in ROS response and defense. [14]

There are other possible explanations for the extreme resistance of this organism to radiation, like its 
protection against prolonging desiccation, nitrous oxide, and S-layer complex. This S-layer Deinoxanthin Binding Complex (SDBC) contributes to its extreme radio resistence. This S-layer acts as a shield against electromagnetic stress, as in the case of ionizing radiation exposure. It also stabilizes the cell wall against possible consequences of high temperature and desiccation. [15-16]

\section{Applications}

In Bioremediation: It refers to a process where microorganisms, fungi, plants, or enzymes are used to restore the contaminated environment to its natural state. The soil, sediments, and water may be contaminated by the nuclear waste in various situations. This contamination may be with a radionuclide, like Uranium, strontium, and cesium or heavy metals like chromium, lead, and mercury, the toxic solvents like Benzene, toluene, xylenes and chlorinated hydrocarbons, etc. The decontamination of such sites poses a real challenge and available cleanup technologies are expensive and dangerous. An alternative is the use of bioremediation with the help of specialized microorganisms that can detoxify the metallic and organic elements and are made harmless to the environment.

The common organisms cannot be used for decontamination because they perish due to high levels of radiation; here $\mathrm{D}$. radiodurans may play a role, since it is known to have resistance to high doses of radiation. It can be used to treat nuclear energy waste. This bacterium ( $\mathrm{D}$. Radiodurans) is engineered genetically to consume and digest the solvents and heavy metals in this radioactive environment. The mercuric reductase gene has been cloned from E.Coli into Dienococcus to detoxify the ionic mercury residue. This residue is a waste generated from the nuclear weapon manufacturing process. [17] These researchers have developed a strain of Dienococcus that could detoxify both mercury and toluene in mixed radioactive waste. A gene encoding a non-specific acid phosphatize from Salmonella enteric serovar Typhi and the alkaline phosphatize gene from Sphingomonas have been introduced in the strains of D.radiodurans for the bio precipitation of uranium in acid and alkaline solutions respectively. The bioengineered $D$. Radiodurans has already established itself as a useful agent to decontaminate radioactive waste sites.

\section{a) Application in Biomedical field}

Aging and cancer are associated with increased DNA and protein oxidation due to ROS generation. There is also a decline in the ability of the cell to protect itself from oxidative damage and to repair the damage of DNA [18]

A focal point of aging and cancer research is to identify factors that antagonize the aging process and carcinogenesis and to design adequate therapeutic strategies. In this field, the bacterium D.radiodurans can be used to study the process of aging and cancer. It is known that the physiological changes that bring about aging and cancer are related to damage to DNA, RNA and, oxidative damage to the cell, thereby weakening its defence and repair mechanism. The D. Radiodurans is known to protect itself from oxidative damages and efficiently repairs its damage to DNA. The same property may be applied to human cells also in the future.

The free radical theory of aging and cancer postulates that the damage caused by the production of ROS is the underlying cause of aging and cancer; Alzheimer's disease and Parkinson's disease are also clearly associated with oxidative stress. Oxidative stress occurs when ROS production is accelerated or when antioxidant defense enzymes are impaired. Oxidative stress affects both DNA and proteins. The efficient repair, therefore is a critical component in the protection against aging and cancer. Since the oxidative stress plays a significant role in the aging and cancer processes, the strategies to combat this process is to reduce the oxidative damage or boost the defense mechanism. The ability of protection of $\mathrm{D}$. radiodurans against oxidative damage can be harnessed to this crucial process, to delay aging and prevent cancer and other age-related diseases. The future researches are directed to employ deinococcal antioxidants to prevent or reduce age- and cancer-related protein modifications and DNA damages.

\section{Discussion}

The Deinococcus Radiodurans is a polyextremophilic bacterium. It can withstand any adverse environmental conditions like cold, heat, desiccation, radioactivity, etc. It is because of multiple copies of genome and exceptional ability to repair the damaged DNA. These unique properties can be used for bioremediation, and biomedical applications. In the field of bioremediation it can be used for decontamination of the toxic environment. This bacterium ( $D$. Radiodurans) is engineered genetically to consume and digest the solvents and heavy metals in radioactive environment. It can also be used to tackle the nuclear energy waste. The D. Radiodurans is known to protect itself from oxidative damages and efficiently repairs its damage to DNA. Cancer and aging cells die due to oxidative damage in human cells. Experiments are going on to study whether the properties of this bacterium can be applied to the human cells also to prevent it from oxidative damages. The ability of protection of $\mathrm{D}$. radiodurans against oxidative damage can be harnessed to this crucial process, to delay aging and prevent cancer and other age-related diseases.

Conflicts of interest: Nil

Funding source: Nil 


\section{References Références Referencias}

1. https://en.wikipedia.org/wiki/Deinococcus radiodur ans accessed on 09.01.2020.

2. Murray, R. G. (1986). Family II. Deinococcaceae, p. 1035-1043. In P. H. A. Sneath, N. S. Mair, M. E. Sharpe, and J. G. Holt (ed.), Bergey's manual of systematic bacteriology, vol. 2. Williams \& Wilkins Co., Baltimore, MD.

3. http://www.genomenewsnetwork.org/articles/07_02/ deinococcus.shtml accessed on 09.01.2020.

4. De Groot A, Chapon V, Servant P, Christen R, Saux MF, Sommer S, Heulin T (2005). "Deinococcus deserti sp. nov., a gamma-radiation-tolerant bacterium isolated from the Sahara Desert". Int J Syst Evol Microbiol. 55 (Pt 6): 2441-2446. doi:10.1099/ijs.0.63717-0. PMID 16280508.

5. Battista, J. R. (1997) "Against all odds: the survival strategies of Dienococcus radiodurans". Annu. Rev. Microbiol. 51:203-224.

6. Anderson, A W; H C Nordan; R F Cain; G Parrish; D Duggan (1956). "Studies on a radio-resistant micrococcus. Isolation, morphology, cultural characteristics, and resistance to gamma radiation". Food Technol. 10 (1): 575-577.

7. Slade, D; Radman, M (2011). "Oxidative stress resistance in Deinococcus radiodurans". Microbiol Mol Biol Rev. 75 (1): 133-91. doi:10.1128/MMBR.00015-10.

8. Moseley BE, Mattingly A (1971). "Repair of irradiated transforming deoxyribonu- cleic acid in wild type and a radiation- sensitive mutant of Micrococcus radiodu- rans". J. Bacteriol. 105 (3): 976-83.

9. Ito $H$, Watanabe $H$, Takeshia M, lizuka H (1983). "Isolation and identification of radiation-resistant cocci belonging to the genus Deinococcus from sewage sludges and animal feeds. Agric". Biol. Chem. 47 (6): 1239-47.

doi:10.1271/bbb1961.47.1239.

10. Daly M. J., Minton K. W. (1996) An alternative pathway of recombination of chromosomal fragments precedes recA-dependent recombination in the radioresistant bacterium Deinococcus radiodurans. J. Bacteriol. 178, 4461-4471.

11. Moseley, BE; Setlow, JK (1968). "Transformation in Micrococcus radiodurans and the ultraviolet sensitivity of its transforming DNA". Proc Natl Acad $\begin{array}{llllll}\text { Sci } U & S & \text { A. } & 61 & \text { (1): 176-183. }\end{array}$ doi:10.1073/pnas.61.1.176.

12. http://cfyn.ifas.ufl.edu/radiation.pdf accessed on 10.01.2020

13. Daly, Michael J.; Elena K. Gaidamakova; Vera Y. Matrosova; Alexander Vasilenko; Min Zhai; Richard D. Leapman, et al (2007). "Protein Oxidation Implicated as the Primary Determinant of Bacterial Radioresistance". PLoS Biology. 5 (4): e92 EP .doi:10.1371/journal.pbio.0050092.
14. Peana M, Chasapis CT, Simula G, Medici S, Zoroddu MA (2018). "A Model for Manganese interaction with Deinococcus radiodurans proteome network involved in ROS response and defense". Journal of Trace Elements in Medicine and Biology. 50: 465-473.

doi:10.1016/j.jtemb.2018.02.001. PMID 29449107.

15. Farci D, Slavov C, Tramontano E, Piano D (2016). "The S-layer Protein DR_2577 BindsDeinoxanthin and under Desiccation Conditions Protects against UV-Radiation inDeinococcus radiodurans" Frontiers in Microbiology. $\quad 155$. doi:10.3389/fmicb.2016.00155

16. Farci D, Slavov C, Piano D (2018). "Coexisting properties of thermo stability and ultraviolet radiation resistance in the main S-layer complex of Deinococcus radiodurans". Photochem Photobiol Sci. 17 (1): 81-88. doi:10.1039/c7pp00240h

17. Brim H, McFarlan SC, Fredrickson JK, Minton KW, Zhai M, Wackett LP, Daly MJ (2000)."Engineering Deinococcus radiodurans for metal remediation in radioactive mixed waste environments" Nature Biotechnology. 18 (1): 85-90. Doi: 10.1038/71986

18. Beckman, K. B., B. N. Ames. (1998) the free radical theory of aging matures. Physiol. Rev. 78:547-581. 